\title{
Recurrent infective endocarditis in a drug addict
}

\author{
Menafra G, Pingitore A*, Gurgo A, Magri D, Pagannone E, Testa M and Volpe M \\ Department of Medicine and Psychology, Sant'Andrea Hospital, Italy
}

\begin{abstract}
Infective endocarditis is a disease with a high mortality rate; mortality increases with the number of new episodes. Patients with relapsing endocarditis have a higher mortality rate per year (20\%) than patients with a single episode (9\%); moreover, the episodes of infective endocarditis on the prosthetic valve have a worse prognosis, with a mortality rate of $45 \%$ for each episode. In relapsing endocarditis, the following are distinguished: the relapses, characterized by the same germ, less than six months after the previous episode, and the reinfections, which may be caused by a different germ, or by the same germ, more than six months after previous episode. A recent analysis of the risk factors of recurrent bacterial endocarditis showed that the use of intravenous drugs, hemodialysis, immunosuppression and previous episodes of infective endocarditis played a major role, especially if they occurred on the prosthetic valve and if caused by $S$. aureus. Recurrent infectious endocarditis occurs more frequently in young male patients with HIV seropositivity, who routinely use intravenous drugs. We have analyzed the clinical case of a 36-year-old immunocompetent man with a long history of parenteral drug abuse, who has presented 6 new episodes of relapsing infective endocarditis on mitral valve and 2 reinfections since 2013. Between 2013 and 2016, the patient underwent two mitral valve replacements with a biological prosthesis implant and a mitral valve replacement with a mechanical prosthesis implant and a tricuspid anuloplasty. The remaining episodes were treated with medical therapy only. Currently in the literature 6 episodes of relapsing infective endocarditis and 1 reinfection in a single patient is the highest number of reported events.
\end{abstract}

\section{Introduction}

Recurrent infective endocarditis (IE) are a relatively common disease in some population of patient, such as IV drug-addicted ones. In the context of recurrent IE we can distinguish: relapse IE, caused by the same bacterial, less than 6 months after the previous episode, and the reinfections, caused by a different bacterial or by the same one, more than 6 months after the previous episode. Multiple episodes of recurrent IE occurred in habitual drug abusers. In the literature about recurrent IE were never reported more than 7 episodes of recurrent IE in a single patient. We describe the extraordinary case of a 32 year old man who presented at our attention for the eighth recurrence of IE.

\section{Case Report}

On July 2017 a 32 years old man with a history of six previous episodes of IE presented at our attention.

The first presentation of IE occurred on February 2013. In that occasion the blood cultures isolated a Staphylococcus aureus multidrug-sensitive. After few days of treatment appeared a cerebral ischemic stroke most likely due to septic embolization hesitated in left hemiparesis. The patient undergone a mitral valve replacement with a biological prosthetic valve.

The second presentation was on January 2015. The blood cultures isolated a Staphylococcus epidermidis. Echocardiography showed a periprosthetic abscess determining a severe mitral regurgitation. Hence the patient undergone an urgent mitral valve replacement with another biological prosthetic valve.

On August 2015 another episode of IE was due to a meticillinresistent Staphylococcus epidermidis and was treated with antibiotic therapy only.

On June 2016 occurred the fourth IE. The Blood cultures isolated a Staphilococcus oralis and was treated with antibiotic therapy only.
On August 2016 the patient was hospitalized for the fifth episode of IE. The blood cultures isolated an Enterococcus faecalis. Echocardiography pointed out severe mitral obstruction due to hyper-echogenic fluctuating images determining the hypomobility of the prosthetic mitral leaflets, mild mitral regurgitation (prosthetic transvalvular mean gradient of $19 \mathrm{mmHg}$ ) and mild intra-prosthetic and double peri-prosthetic mitral regurgitation. During the hospitalization occurred an episode of acute heart failure, treated successfully with medical therapy.

Unfortunately on November 2016 another IE occurred. The blood cultures isolated the same antibiotic-sensibility spectrum Enterococcous faecalis. During this hospitalization the damaged biological prosthetic mitral valve was substituted with a mechanical prosthetic mitral valve (Saint Jude Medical $31 \mathrm{~mm}$ ); moreover a tricuspid valve annuloplastic (De Vega technique) was performed because of significant regurgitation due to annular dilatation.

On July 2017 the patient has accessed to the Emergency Room of our Hospital with a fever up to $39^{\circ} \mathrm{C}$. The blood cultures isolated a Staphilococcus aureus. The transesophageal echocardiography documented a fluctuating vegetation $(15 \mathrm{~mm} \times 5 \mathrm{~mm})$ on the lateral portion of the mitral prosthesis ring, apparently not affecting the dynamics of the emi-discs (Figure 1).

The case was presented to the cardiac surgeons and collectively discussed. Taken into account the very high surgical risk, once informed the patient, a new mitral valve replacement intervention was not indicated and was chosen a conservative strategy based on

${ }^{\star}$ Correspondence to: Pingitore A, Department of Medicine and Psychology, Sant'Andrea Hospital, Italy, Tel: +39 06 33771, Email: annachiara.pingitore@ gmail.com

Received: April 20, 2018; Accepted: April 27, 2018; Published: April 30, 2018 
the already ongoing antibiotic therapy. For the sudden onset of left hemiplegia, patient undergone to cerebral RM which documented recent ischaemic lesion in the right-capsule lens (Figure 2).

Therefore was started therapy with linezolid, meropenem and fluconazole in addition to vancomycin in suspicion of brain abscess. In pre-discharge the antibiotic therapy with imipenem and linezolid was suspended and was started the somministration of amoxicillin/ clavulanic acid.

One month later, after a new episode of drug abuse, the patient presented a fever up to $39^{\circ} \mathrm{C}$. The patient came to our Emergency

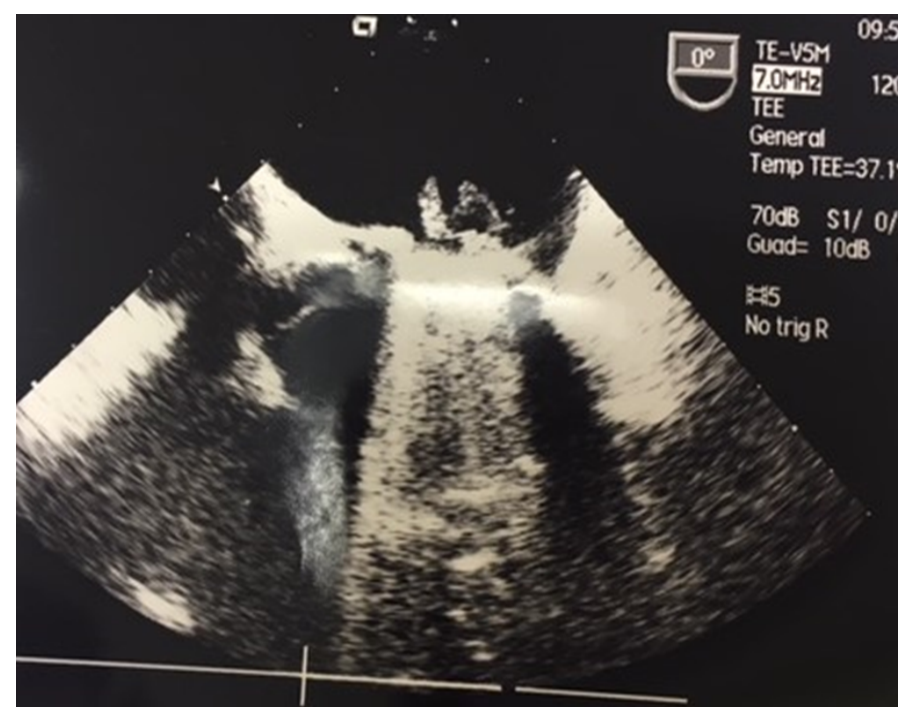

Figure 1. A fluctuating vegetation $(15 \mathrm{~mm} \times 5 \mathrm{~mm})$ on the lateral portion of the mitral prosthesis ring.

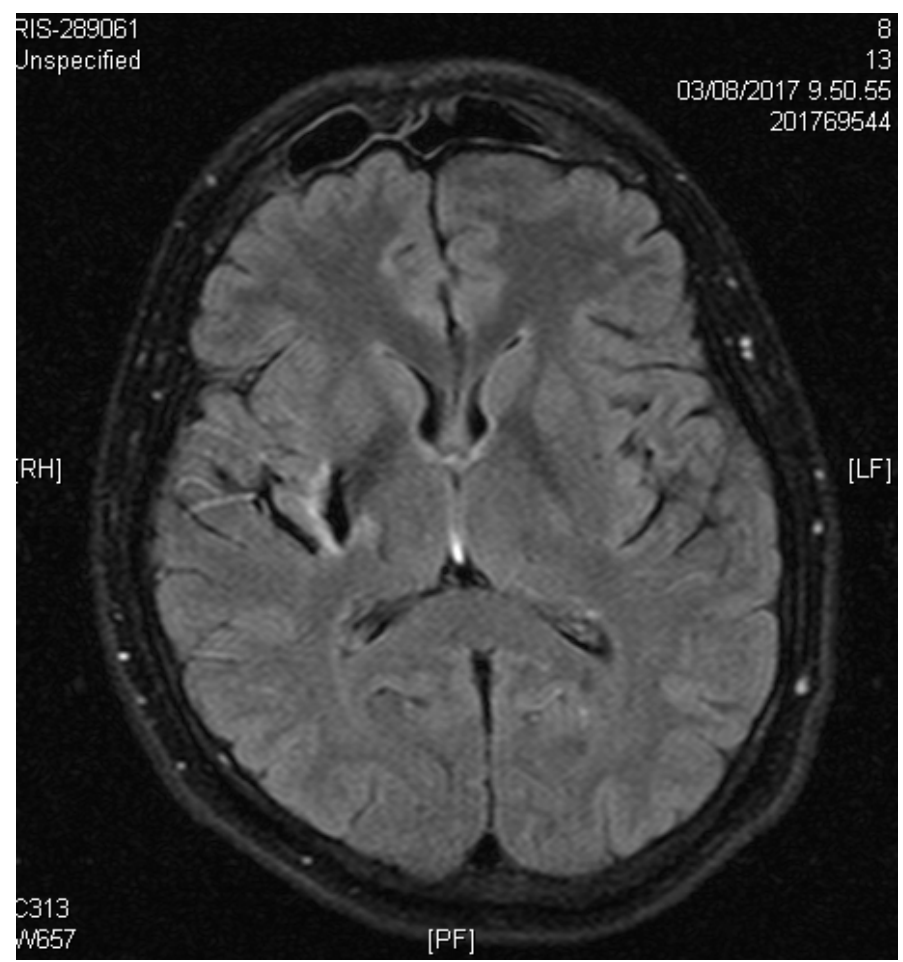

Figure 2. A thromboembolic ischaemic lesion in the right-capsule lens caused by endocardial vegetation.
Room hemodynamically unstable (undetectable blood pressure, FC $110 \mathrm{bpm}$ ). The ECG showed T waves negative-difasic in the anterior leads, QTc $491 \mathrm{msec}$. After few ours occurred a cardiac arrest on ventricular fibrillation, promptly treated with DC shock. Toxicological tests resulted positive for cocaine, tetrahydrocannabinol and benzodiazepine. The patient was admitted to the Coronary Intensive Therapy. The blood cultures isolated Staphilococcus aureus for which a specific therapy, according to the antibiogram, was set (gentamicin and vancomycin) with improvement of the laboratory and clinical conditions. Therefore, the patient was moved to the Cardiology ward. Transesophageal echocardiogram showed at the level of the mitral mechanical prosthesis some images in plus, mobile, of maximum dimensions of $0.6 \times 0.4 \mathrm{~cm}$ (likely residuals of the previous endocarditis) in the absence of significant transvalvular gradient. During the hospitalization, there was a recurrence of fever up to $40^{\circ} \mathrm{C}$ and, at the same time, a new increase in the inflammation indexes was observed. New blood cultures resulted positive for multi-drug resistant (MDR) Klebsiella pneumoniae. Antibiotic therapy was initially modified starting Oxacillin and Daptomycin and, subsequently, according to the antibiogram, Phosphomicin was added without apparent not clinical neither laboratory parameters improvement. Another blood sample was sent to the laboratory; because of further positivity of blood cultures for MDR Klebsiella Pneumoniae, antibiotic therapy with Tigecycline and Colimycin was introduced in association with Fosfomycin, suspending Daptomycin and Oxacillin. The patient did not show fever again with complete normalization of the inflammatory indexes. The blood cultures were performed again resulting negative. Trans-esophageal echocardiogram was repeated and showed at the level of the mechanical mitral prosthesis filamentous image of about $0,6 \times 0,3 \mathrm{~cm}$ (in the first instance attributed to outcomes) with an average prosthetic gradient of $8 \mathrm{mmHg}$ and 2 small regurgitation jets, determining a slight insufficiency. The patient was discharged in good clinical conditions.

\section{Discussion}

Endocarditis is a high-rate mortality disease. Patients with repeat IE have higher one-year mortality rate (20\%) than those with a single episode (9\%) [1]; moreover the IE episodes involving a prosthetic valve have a worse prognosis reaching the mortality rate of $45 \%$ per single episode. In a recent analysis of risk factors of repeat endocarditis emerged that injection drug use, previous infective endocarditis episode, Staphylococcus aureus IE, haemodialysis, healthcare acquisition and HIV infection were at high risk of developing recurrent IE [2]. Habitual drug abuse is such an important risk factor of IE and recurrent IE that was even included in the minor diagnostic criteria (modified Dukes criteria) of last IE ESC guidelines (2015) [3]. Male predominance is historically documented, this is partially due to the contribute of drug addicted population which is mainly composed by this gender [4]. The patient that we presented was a immunocompetent 32 years-old male at the moment of his first IE episode, with long history of injection drug abuse. Our patient presented a total of 8 IE episodes, of which 6 new documented infections ( $>6$ months passed from the previous episode and/or different responsible bacterium) [5]; all the episodes involved the mitral valve over a period of 4 years. Furthermore, two episodes were complicated by ischaemic strokes caused by cerebral septic embolization, a clinic condition that got the patient's prognosis even worse [6]. The huge number of relapses is peculiar, given the high one-year mortality rate of the disease; another remarkable feature is the exclusive mitral valve involvement, although the occurrence of right-heart endocarditis in parenteral drug-addicted patients is very common to observe $[7,8]$. In addition, the patient undergone to two biological prosthetic valve and one mechanical 
prosthetic valve implantations, with an increasing operative mortality risk at every surgery [9]. The first episode occurred on a healthy heart native valve, showing that the intravenous drug abuse represents an independent risk factor for IE. The following 6 episodes involved the biological prosthetic valves over 20 months and just the last 2 episodes affected the mechanical prosthetic valve over 10 months; this proves that a prosthetic valve implantation, independently from the valve type, represents an important risk factor for recurrent IE [10]. Additionally, we must highlight the relevant amount of relapse episodes despite the patient had no immunocompromission and was not using intravenous drugs since one year. In summary, this case report demonstrates that some risk factors hardly impact on the relapse number of IE; certainly the young age and the immunocompetence contributed to so many relapse episodes survival.

\section{References}

1. Slipczuk L, Codolosa JN, Davila CD, Romero-Corral A, Yun J et al. (2013) Infective endocarditis epidemiology over five decades: a systematic review. PLoS One 8: e82665. [Crossref]

2. Alagna L, Park LP, Nicholson BP, Keiger AJ, Strahilevitz J, et al. (2014) Repeat endocarditis: analysis of risk factors based on the International Collaboration on Endocarditis - Prospective Cohort Study. Clin Microbiol Infect 20: 566-575. [Crossref]
3. (2015) Modified Dukes criteria - IE ESC guidelines.

4. Vogkou CT, Vlachogiannis NI, Palaiodimos L, Kousoulis AA (2016) The causative agents in infective endocarditis: a systematic review comprising 33,214 cases. Eur J Clin Microbiol Infect Dis 35: 1227-1245. [Crossref]

5. Chu VH, Sexton DJ, Cabell CH, Reller LB, Pappas PA, et al. (2005) Repeat infective endocarditis: differentiating relapse from reinfection. Clin Infect Dis 41: 406-9. [Crossref]

6. Ruttmann E, Willeit J, Ulmer H, Chevtchik O, Höfer D, et al. (2006) Neurologica outcome of septic cardioembolic stroke after infective endocarditis. Stroke 37: 20942099. [Crossref]

7. Sousa C, Botelho C, Rodrigues D, Azeredo J, Oliveira R (2012) Infective endocarditis in intravenous drug abusers: an update. Eur J Clin Microbiol Infect Dis 31: 2905-2910. [Crossref]

8. Miró JM, del Río A, Mestres CA (2002) Infective endocarditis in intravenous drug abusers and HIV-1 infected patients. Infect Dis Clin North Am 16: 273-295, vii-viii. [Crossref]

9. Fedoruk LM, Jamieson WR, Ling H, Macnab JS, Germann E, et al. (2009) Predictors of recurrence and reoperation for prosthetic valve endocarditis after valve replacement surgery for native valve endocarditis. J Thorac Cardiovasc Surg 137: 326-33. [Crossref]

10. Tao E, Wan L, Wang W, Luo Y, Zeng J, et al. (2017) The prognosis of infective endocarditis treated with biological valves versus mechanical valves: A meta-analysis PLoS One 12: e0174519. [Crossref]

Copyright: (C2018 Menafra G. This is an open-access article distributed under the terms of the Creative Commons Attribution License, which permits unrestricted use, distribution, and reproduction in any medium, provided the original author and source are credited. 